\title{
Theory of Raman-Mediated Pulsed Amplification in Silicon-Wire Waveguides
}

\author{
Xiaogang Chen, Nicolae C. Panoiu, and Richard M. Osgood, Jr., Fellow, IEEE
}

\begin{abstract}
We present a comprehensive theoretical study of pulsed stimulated Raman scattering in silicon wires. The pulse dynamics is described by a system of coupled equations, which describes intrinsic waveguide optical losses, phase shift and losses due to free-carriers (FCs) generated through two-photon absorption (TPA), first- and second-order frequency dispersion, self-phase and cross-phase modulation, TPA losses, and the interpulse Raman interaction. Furthermore, the influence of the FCs on the pulse dynamics is incorporated through a rate equation. The corresponding system of equations has then been numerically integrated, and phenomena such as noise-seeded Raman amplification, pulsed Raman amplification, and Raman-mediated pulse interaction have been described.
\end{abstract}

Index Terms-Coupled mode analysis, Raman amplification, silicon nanowire waveguide, stimulated Raman scattering (SRS).

\section{INTRODUCTION}

$\mathbf{R}$ ECENTLY, active silicon-waveguide components have attracted much interest for use in photonic integrated circuits. Due to its indirect band, direct optical pumping cannot be employed to realize light amplification in silicon. However, several groups have shown recently that stimulated Raman scattering (SRS) can be used to achieve tunable light amplification in the C-band communication channel. Experiments have been performed to prove the validity of this idea using silicon-on-insulator (SOI) waveguides [1], [2] or SOI wire waveguides with submicron transverse dimensions [3], [4]. The feasibility of employing silicon-waveguides in active devices has been further demonstrated by the recent fabrication of SRS-based silicon lasers [5]-[8] and frequency converters [9], [10].

In these recent experiments, the cross section of the waveguides ranged from a few [7], [8] to tens of square microns [5]. For these waveguides, the material dispersion dominates. On the other hand, SRS amplification has also been demonstrated in a SOI wire waveguide [3], which had a cross section of less than $0.1 \mu \mathrm{m}^{2}$. In such a waveguide, the light confinement is strongly enhanced, and, therefore, higher power densities can be achieved. As a result, Raman amplification is achieved at smaller optical powers and unwanted effects such as free-carrier (FC) generation and two-photon absorption (TPA) are reduced. An additional advantage of using such ultra-small wire waveguides stems from the fact that for waveguides with submicron transverse dimensions the waveguide dispersion dominates the

Manuscript received July 15, 2005; revised October 12, 2005. This work was supported by DARPA/MTO University Opto Centers under Contract BROWNU-1119-24596, DoD STTR Grant FA9550-04-C-0022, and by AFOSR Grant FA9550-05-1-0428.

The authors are with Microelectronics Sciences Laboratories, Columbia University, New York, NY 10027 USA (e-mail: xiaogang.chen@columbia.edu).

Digital Object Identifier 10.1109/JQE.2005.861827 material dispersion, and, therefore, the total modal dispersion can be easily engineered.

All of these SRS experiments have used high pump power density and a variety of pump formats; hence, it is crucial to have a high-intensity theoretical model that describes the influence of optical nonlinearities on pulse dynamics in the presence of Raman interaction. We present here a rigorous analysis, based on the coupled-mode formalism, of pulsed SRS in silicon wire waveguides. Dispersive effects, which are absent in the continuous pump case, play an important role in the Raman-mediated pulse interaction and Stokes amplification. Free carrier and TPA effects contribute to additional loss in the waveguide and are thus incorporated in our study. The magnitude of the effective third-order nonlinearity is sensitive to the waveguide propagating-mode profile, due to the tight mode confinement within the SOI waveguide, and is included in our model through several scalar coefficients, set by overlap integrals. Our model can be easily integrated numerically, and, therefore, offers a fast route to a detailed analysis of the Raman-mediated pulsed amplification in silicon wires.

The paper is organized as follows. In the next section we describe the dispersive properties of the silicon wire waveguides and discuss the relative contribution of the material and waveguide dispersion to the modal dispersion. Section III is devoted to the formulation of the analytical model that governs the combined dynamics of the optical field and the FCs. The corresponding system of equations is integrated numerically and the results of these detailed numerical studies are presented in Section IV. Finally, we summarize our results in Section V.

\section{Dispersive Properties OF Si Wire WAVEGUIDES}

The schematics of the structure of the silicon wire waveguide considered here is presented in Fig. 1. It shows a rectangular silicon core oriented along the [110] direction, which is embedded into a $\mathrm{SiO}_{2}$ substrate whose refractive index is $n_{s}=$ 1.45 at $1.55 \mu \mathrm{m} \mathrm{[3].} \mathrm{Both} \mathrm{the} \mathrm{width} w$ and height $h$ of the silicon core are in the submicron range. The main consequence of this choice of the geometrical parameters of the wire waveguide is that, unlike the case of the waveguides used in recent experiments [1], [5], [7], [8], the waveguide dispersion dominates the material dispersion. As we will demonstrate, this property plays an important role in defining the pulse dynamics in the presence of Raman interaction. Although our analysis applies to waveguides with arbitrary submicron dimensions, most of the quantitative results reported here are calculated by using the parameter values $w=0.45 \mu \mathrm{m}$ and $h=0.22 \mu \mathrm{m}$, which correspond to the silicon wire used by the authors of [3] to demonstrate Raman amplification in ultrasmall SOI wire waveguides [3]. 

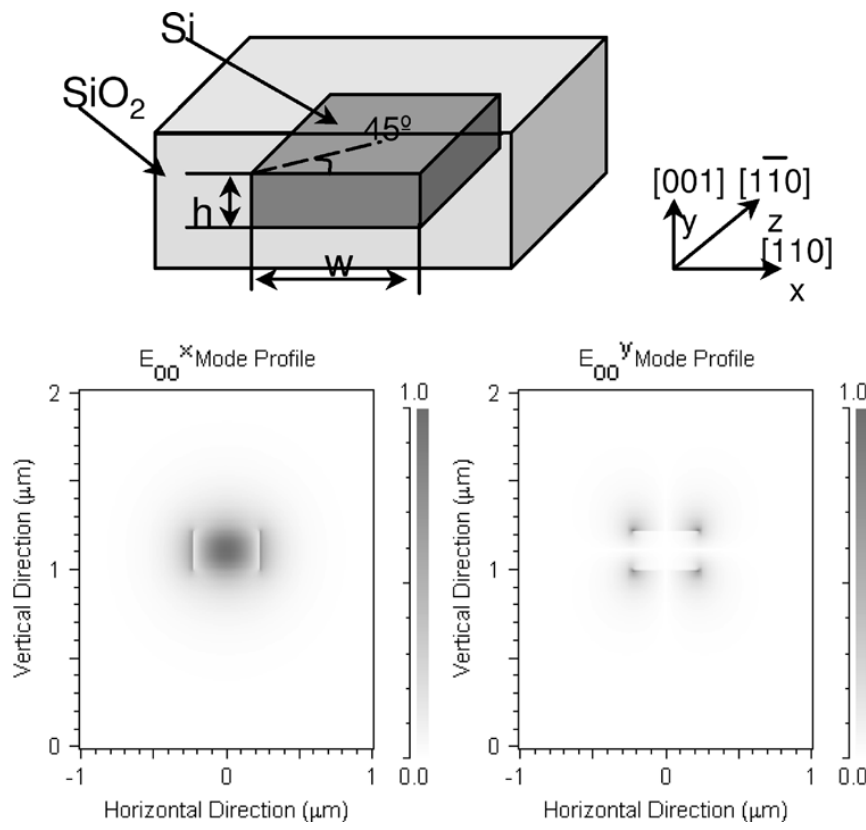

Fig. 1. Waveguide geometry of the Si wire, with the input facet of the waveguide in the $(1 \overline{1} 0)$ plane of the Si crystal lattice. The dashed-line is along the [100] direction. The waveguide width and height are $w$ and $h$, respectively.

The dispersive properties of four silicon wire waveguides with different dimensions are illustrated in Fig. 2. The values chosen for the geometrical parameters $(w, h)$ were, respectively, $(0.45 \mu \mathrm{m}, 0.22 \mu \mathrm{m}),(0.55 \mu \mathrm{m}, 0.22 \mu \mathrm{m})$, $(0.65 \mu \mathrm{m}, 0.22 \mu \mathrm{m})$, and $(0.45 \mu \mathrm{m}, 0.3 \mu \mathrm{m})$. The guiding modes were computed by using BeamPROP ${ }^{1}$ a commercially available software tool based on the fully-vectorial implementation of the beam propagation method. In all these cases the waveguides support only one mode, namely the $E_{11}^{x}$ mode, whose main components of the electromagnetic field are $E_{x}$ and $H_{y}$. Because of the high-index contrast provided by the SOI materials platform, as well as the subwavelength transverse dimensions of the waveguide, the guided modes exhibit strong frequency dispersion. For example, Fig. 2(a) shows that the waveguide dispersion in such wire waveguides dominates the material dispersion. The contribution of the latter to the mode dispersion is given by the relation $\beta_{m}=2 \pi n(\lambda) / \lambda$, where $\beta_{m}$ is the propagation constant, $\lambda$ is the wavelength, $c$ is the speed of light, and the material refractive index $n(\lambda)$ is given by the Sellmeier equation [11]

$$
n^{2}(\lambda)=\epsilon+\frac{A}{\lambda^{2}}+\frac{B \lambda_{1}^{2}}{\lambda^{2}-\lambda_{1}^{2}} .
$$

For silicon, the material constants in (1) are $\lambda_{1}=1.1071 \mu \mathrm{m}$, $\epsilon=11.6858, A=0.939816 \mu \mathrm{m}^{2}$, and $B=8.10461 \times 10^{-3}$. The first two terms represent the nonresonant contributions to the refractive index, whereas the last one gives the contribution of the indirect band-gap.

The total modal dispersion, which includes both the waveguide and the material dispersion, is used then to numerically calculate the higher-order dispersion coefficients, defined by the relation $\beta_{i}(\omega)=\partial^{i} \beta(\omega) / \partial \omega^{i}$ [12], where $\omega$ is the frequency

${ }^{1}$ RSoft Design Group. [Online] Available: http://www.rsoftdesign.com/
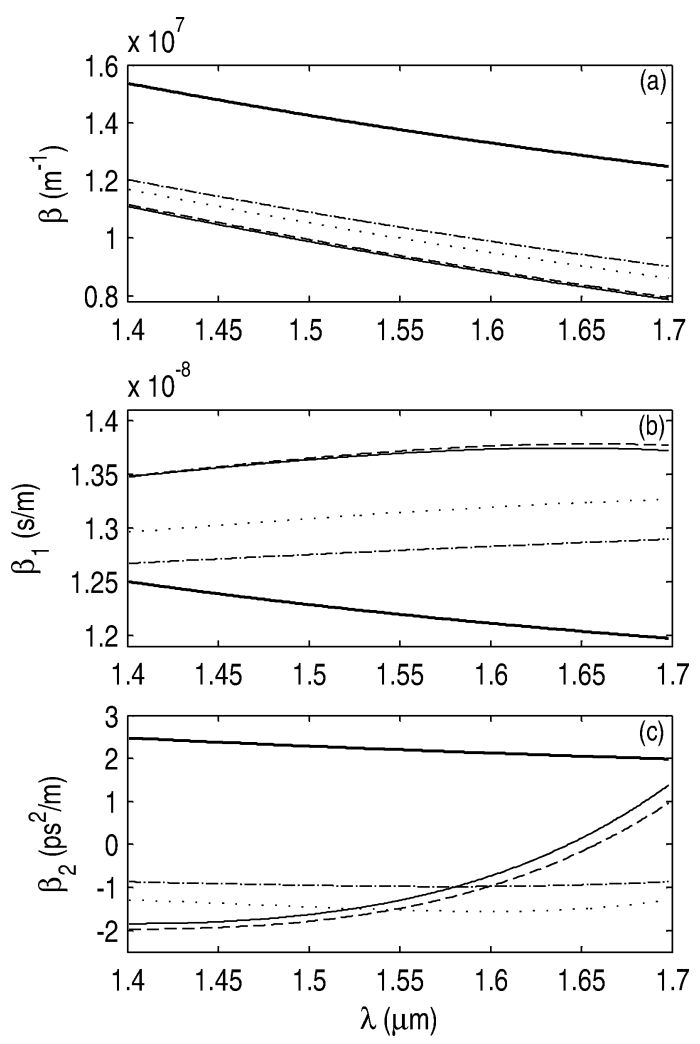

Fig. 2. Dispersion coefficients for several nanowire waveguides. (a) Propagation constant versus wavelength. (b) First-order dispersion coefficient versus wavelength. (c) Second-order dispersion coefficient versus wavelength. In all the figures, the thick lines represent the material dispersion, whereas the dispersion data correspond to the waveguides with dimensions of $(0.45 \mu \mathrm{m}, 0.22 \mu \mathrm{m})(-) ;(0.45 \mu \mathrm{m}, 0.30 \mu \mathrm{m})(---) ;(0.55 \mu \mathrm{m}, 0.22 \mu \mathrm{m})$ $(\cdots)$; and $(0.65 \mu \mathrm{m}, 0.22 \mu \mathrm{m})(-\cdot-\cdot-)$.

and $i$ is the corresponding order. The results of these calculations are presented in Fig. 2(b) and Fig. 2(c), which show, respectively, the first- and second-order dispersion coefficients. One important property illustrated in these figures is that by engineering the waveguide modal dispersion one can design waveguides that exhibit both normal $\left(\beta_{2}>0\right)$ and anomalous dispersion $\left(\beta_{2}<0\right)$ within the same frequency window of important technological applications. For completeness, we have also calculated the third-order dispersion coefficient, but as we will explain later the corresponding third-order dispersion plays a negligible role on the dynamics of the Raman mediated pulse interaction.

\section{THEORETICAL MODEL OF RAMAN MEDIATED PULSE INTERACTION}

The main characteristics of the dynamics of the Raman-mediated pulse interaction in a silicon wire are governed by the interplay between several characteristic times and the relative magnitude of several characteristic lengths. Thus, if a monochromatic pump wave at frequency $\omega_{p}$ propagates in a Raman-active solid medium it creates a scattered Stokes signal at the frequency $\omega_{s}$, which is down-shifted with respect to $\omega_{p}$ by a frequency shift $\omega_{R}$ (Raman frequency) that corresponds to the frequency of the optical phonons at the center of the Brillouin zone. For silicon, this frequency shift 
is $\omega_{R} / 2 \pi=\sim 15.6 \mathrm{THz}$ [13]. Furthermore, in silicon the spectral width of the SRS spectrum is $\Delta \omega / 2 \pi=\sim 105 \mathrm{GHz}$, which amounts to a response time of the Raman interaction of $\tau=\sim 10 \mathrm{ps}$. Therefore, if the pulsewidth of the pump and Stokes pulses are much larger than the Raman response time $\tau$, their spectral width is much smaller than the width of the Raman spectrum, so that the Raman interaction is mediated by the third-order nonlinear Raman susceptibility, calculated exactly at the frequency $\omega_{R}$. In the opposite case, namely when the pulsewidth of the pump and Stokes pulses are comparable or smaller than the Raman response time, all frequencies in the Raman spectrum are involved in the process of energy transfer from the pump to the Stokes pulse.

In addition to the characteristic time of Raman interaction, there are also three characteristic lengths that play an important role in the stimulated Raman amplification of the Stokes signal. These are the waveguide length $L$, and the walkoff and dispersion lengths defined, respectively, as

$$
\begin{aligned}
L_{w} & =\frac{T_{0 p}}{\left|d_{p s}\right|} \\
L_{d} & =\frac{T_{0 p}^{2}}{\left|\beta_{2 p}\right|}
\end{aligned}
$$

where $T_{0 p}$ is the half-width (at $1 / e$-intensity point) of the pump pulse and $d_{p s}=\beta_{1 p}-\beta_{1 s}=1 / v_{g p}-1 / v_{g s}$ is the walkoff parameter, with the group-velocity $v_{g}=1 / \beta_{1}$. For a pump pulse with temporal width $T_{0 p}=5 \mathrm{ps}$, centered at $\lambda_{p}=1.55 \mu \mathrm{m}$, and the corresponding Stokes pulse centered at $\lambda_{s}=1.67 \mu \mathrm{m}$ the propagation constants are $\beta_{1 p}=1.3695 \times 10^{-8} \mathrm{~s} / \mathrm{m}$ and $\beta_{1 s}=1.3737 \times 10^{-8} \mathrm{~s} / \mathrm{m}$, respectively, so that the characteristic lengths are $L_{w}=11.9 \mathrm{~cm}, L_{d}=19.27 \mathrm{~m}$, and the walkoff parameter is $d_{p s}=-4.2 \times 10^{-11} \mathrm{~s} / \mathrm{m}$. The walkoff length $L_{w}$ represents the distance over which the pump and Stokes pulses pass through each other's envelope, which means that the efficiency of the Raman amplification process increases with $L_{w}$, as long as the condition $L_{w}>L$ is satisfied.

In order to derive the system of equations that governs the dynamics of the Raman mediated pulse interaction in a silicon wire waveguide we start from the conjugated form of the Lorentz reciprocity theorem (see, e.g., [14])

$$
\frac{\partial}{\partial z} \int_{A_{\infty}} \mathbf{F}_{c} \cdot \hat{\mathbf{e}}_{z} d A=\int_{A_{\infty}} \nabla \cdot \mathbf{F}_{c} d A
$$

where the integral is taken over the infinite cross section $A_{\infty}$ of the waveguide, $\hat{\mathbf{e}}_{z}$ is the unit vector oriented along the longitudinal axis of the waveguide, and the vector field $\mathbf{F}_{c}$ is constructed with two arbitrary guided electromagnetic fields $\left(\mathbf{E}_{1}\right.$, $\left.\mathbf{H}_{1}\right)$ and $\left(\mathbf{E}_{2}, \mathbf{H}_{2}\right)$

$$
\mathbf{F}_{c}=\mathbf{E}_{1}^{*} \times \mathbf{H}_{2}+\mathbf{E}_{2} \times \mathbf{H}_{1}^{*} .
$$

Here we assume that both the electric and magnetic fields contain the implicit harmonic time dependence $\exp (-i \omega t)$. Now let us choose the two guided electromagnetic fields to be $\left(\mathbf{E}_{1}, \mathbf{H}_{1}\right)=\left(\mathbf{E}_{0}, \mathbf{H}_{0}\right)$ and $\left(\mathbf{E}_{2}, \mathbf{H}_{2}\right)=(\tilde{\mathbf{E}}, \tilde{\mathbf{H}})$, where the fields

$$
\begin{aligned}
\mathbf{E}_{0}(\mathbf{r} ; \omega) & =\mathbf{e}\left(\mathbf{r}_{t} ; \omega\right) e^{i \beta(\omega) z} \\
\mathbf{H}_{0}(\mathbf{r} ; \omega) & =\mathbf{h}\left(\mathbf{r}_{t} ; \omega\right) e^{i \beta(\omega) z}
\end{aligned}
$$

correspond to the guided mode of the unperturbed lossless waveguide whereas the fields $(\tilde{\mathbf{E}}, \tilde{\mathbf{H}})$ correspond to the waveguide whose optical properties are perturbed both by the nonlinear optical effects such as the SRS, Kerr effect, or the TPA, as well as by the changes of the dielectric constant induced by the linear losses or free carrier generation. Note that the $(\tilde{\mathbf{E}}$, $\tilde{\mathbf{H}})$ fields correspond to a fixed frequency $\omega$, and, therefore, they represent the Fourier components of the time-dependent fields propagating in the perturbed waveguide. In (6) $\mathbf{r}_{t}$ is the transverse coordinate, and $\mathbf{e}\left(\mathbf{r}_{t} ; \omega\right)$ and $\mathbf{h}\left(\mathbf{r}_{t} ; \omega\right)$ are the electric and magnetic fields of the unperturbed mode with frequency $\omega$, normalized such that

$$
\frac{1}{2} \int_{A_{\infty}}\left(\mathbf{e} \times \mathbf{h}^{*}\right) \cdot \hat{\mathbf{e}}_{z} d A=\frac{1}{2} \int_{A_{\infty}}\left(\mathbf{e}^{*} \times \mathbf{h}\right) \cdot \hat{\mathbf{e}}_{z} d A=\mathcal{N} .
$$

Here $\mathcal{N}$ is a normalization constant that defines the mode optical power, $P=|a|^{2} \mathcal{N}$, where $a$ is the mode amplitude.

With this choice of the two electromagnetic fields, (4) can be written as

$$
\frac{\partial}{\partial z} \int_{A_{\infty}}\left[\mathbf{E}_{0 t}^{*} \times \tilde{\mathbf{H}}_{t}+\tilde{\mathbf{E}}_{t} \times \mathbf{H}_{0 t}^{*}\right] \cdot \hat{\mathbf{e}}_{z} d A=i \omega \int_{A_{\infty}} \delta \tilde{\mathbf{P}} \cdot \mathbf{E}_{0}^{*} d A
$$

where the subscript $t$ signifies the transverse component of the field. On the left-hand side of this equation, only the transverse components of the fields are retained as the longitudinal components do not contribute to the integrand. Using Maxwell's equations, the divergence of the vector field $\mathbf{F}_{c}$ leads to the integrand on the right hand side. $\delta \tilde{\mathbf{P}}(\mathbf{r} ; \omega)=\delta \epsilon(\mathbf{r} ; \omega) \tilde{\mathbf{E}}(\mathbf{r} ; \omega) \equiv \delta \tilde{\mathbf{P}}_{\text {lin }}(\mathbf{r} ; \omega)+\delta \tilde{\mathbf{P}}_{\mathrm{nl}}(\mathbf{r} ; \omega)$ is the perturbed polarization, with $\delta \tilde{\mathbf{P}}_{\text {lin }}(\mathbf{r} ; \omega)$ and $\delta \tilde{\mathbf{P}}_{\text {nl }}(\mathbf{r} ; \omega)$ its linear and nonlinear components, respectively. The source of this perturbed polarization is the change in the dielectric constant, $\delta \epsilon(\mathbf{r} ; \omega)$, induced by both linear processes, such as optical losses and free carrier generation, as well as nonlinear optical effects, such as TPA, SRS, and the Kerr effect.

Now let us consider the total time-dependent electric and magnetic fields in the waveguide, written as a superposition of the copropagating pump and Raman pulses

$$
\begin{aligned}
\mathbf{E}(\mathbf{r}, t) & =\mathbf{E}\left(\mathbf{r}, t ; \omega_{p}\right) e^{-i \omega_{p} t}+\mathbf{E}\left(\mathbf{r}, t ; \omega_{s}\right) e^{-i \omega_{s} t}+\text { c.c. } \\
\mathbf{H}(\mathbf{r}, t) & =\mathbf{H}\left(\mathbf{r}, t ; \omega_{p}\right) e^{-i \omega_{p} t}+\mathbf{H}\left(\mathbf{r}, t ; \omega_{s}\right) e^{-i \omega_{s} t}+\text { c.c. }
\end{aligned}
$$

where $\mathbf{E}\left(\mathbf{r}, t ; \omega_{p, s}\right)$ and $\mathbf{H}\left(\mathbf{r}, t ; \omega_{p, s}\right)$ are slowly varying amplitudes of the electric and magnetic fields at the corresponding frequencies, respectively, and c.c. stands for complex conjugation. Note that the field representation (9) is valid as long as the two pulses are well separated in frequency. For Raman interaction in silicon this requirement is always satisfied because of the large value of the Raman frequency $\omega_{R}$ and the relatively small Raman spectral bandwidth $\Delta \omega$. Thus, the field representation (9) ceases to be valid when the pump and the Raman pulses start to spectrally overlap; however, this can happen only for subpicosecond pulses, that is for pulses with a duration considerably smaller than the Raman response time of silicon.

Under our assumption that the frequency bandwidth of the pump and Raman pulses is smaller than the spectral width of the SRS spectrum, it requires that the spectral bandwidths of the envelopes $\mathbf{E}\left(\mathbf{r}, t ; \omega_{p, s}\right)$ and $\mathbf{H}\left(\mathbf{r}, t ; \omega_{p, s}\right)$ are smaller than 
$\Delta \omega$. Furthermore, within the so-called improved coupled-mode analysis formalism [15]-[18] the envelopes of the electric and magnetic fields can be written as

$$
\begin{aligned}
\mathbf{E}_{t}\left(\mathbf{r}, t ; \omega_{j}\right) & =u_{j}(z, t) \mathbf{E}_{0 t}\left(\mathbf{r} ; \omega_{j}\right) \\
\mathbf{H}_{t}\left(\mathbf{r}, t ; \omega_{j}\right) & =u_{j}(z, t) \mathbf{H}_{0 t}\left(\mathbf{r} ; \omega_{j}\right) \\
\mathbf{E}_{z}\left(\mathbf{r}, t ; \omega_{j}\right) & =u_{j}(z, t) \frac{\epsilon^{0}\left(\mathbf{r}_{t}\right)}{\epsilon(\mathbf{r})} \mathbf{E}_{0 z}\left(\mathbf{r} ; \omega_{j}\right) \\
\mathbf{H}_{z}\left(\mathbf{r}, t ; \omega_{j}\right) & =u_{j}(z, t) \mathbf{H}_{0 z}\left(\mathbf{r} ; \omega_{j}\right)
\end{aligned}
$$

where $u_{j}(z, t)$ are slowly varying functions, in both $z$ and $t$, and $j=p, s$. With the normalization (7), the optical powers of the pump and Raman pulses are $\left|u_{p}(z, t)\right|^{2} P_{p}$, and $\left|u_{s}(z, t)\right|^{2} P_{s}$, respectively, where $P_{p}$ and $P_{s}$ are the corresponding peak powers. Note that if one considers a more complex waveguide geometry, namely arrays of strongly coupled wire waveguides, then a fully-vectorial coupled-mode analysis must be employed [19], in which case the transverse fields also depend on the transverse gradient of the longitudinal fields. However, for our case (10) provides an accurate representation of the electromagnetic field distribution in the perturbed waveguide. Moreover, the longitudinal components (10c) and (10d) of the modal fields have been presented only for the sake of completeness, as they represent less than $1 \%$ of the total field amplitude. By inserting (6), (9), and (10) in (8), one obtains

$i \frac{\partial}{\partial z} \sum_{j=p, s} \tilde{u}_{j}\left(z, \omega-\omega_{j}\right) e^{-i\left[\beta(\omega)-\beta\left(\omega_{j}\right)\right] z}=-\frac{\omega}{4 \mathcal{N}} \int_{A_{\infty}} \delta \tilde{\mathbf{P}} \cdot \mathbf{E}_{0}^{*} d A$

where $\tilde{u}_{j}(z, \omega)$ is the Fourier transform of $u_{j}(z, t)$

$$
\tilde{u}_{j}(z, \omega)=\int_{-\infty}^{\infty} u_{j}(z, t) e^{i \omega t} d t
$$

Expanding in Taylor series the difference in the modal propagation constant $\beta(\omega)-\beta\left(\omega_{j}\right)$, the lift-hand side (1.h.s) of (11) becomes

$$
\begin{aligned}
\text { l.h.s. }=\sum_{j=p, s}\{ & i \frac{\partial \tilde{u}_{j}\left(z, \omega-\omega_{j}\right)}{\partial z} \\
& +\left[\beta_{1 j}\left(\omega-\omega_{j}\right)+\frac{1}{2} \beta_{2 j}\left(\omega-\omega_{j}\right)^{2}+\ldots\right] \\
& \left.\times \tilde{u}_{j}\left(z, \omega-\omega_{j}\right)\right\} e^{-i\left[\beta(\omega)-\beta\left(\omega_{j}\right)\right] z}
\end{aligned}
$$

Note that in the series expansion in (13) we neglect the terms beyond the second-order dispersion, as our numerical calculations show that for pulsewidths considered in this paper, the third-order dispersion is at least one order of magnitude smaller as compared to the second-order dispersion. For instance, for a 50-ps pulse centered at $1.55 \mu \mathrm{m}$, the second order dispersion length $L_{d}=19.27 \mathrm{~m}$, while the third order dispersion length $L_{d}^{\prime}=T_{0}^{3} /\left|\beta_{3 p}\right|=11.18 \mathrm{~km}$.

The linear component of the polarization $\delta \tilde{\mathbf{P}}(\mathbf{r} ; \omega)$ in (11) is determined by the linear change in the dielectric constant, $\delta \tilde{\mathbf{P}}_{\operatorname{lin}}(\mathbf{r} ; \omega)=\delta \epsilon_{\operatorname{lin}}(\mathbf{r} ; \omega) \tilde{\mathbf{E}}(\mathbf{r} ; \omega)$, which can be written as

$$
\delta \epsilon_{\operatorname{lin}}(\mathbf{r} ; \omega)=\frac{i \epsilon_{0} c n \alpha_{\mathrm{in}}}{\omega}+2 \epsilon_{0} n \delta n_{\mathrm{FC}}+\frac{i \epsilon_{0} c n \alpha_{\mathrm{FC}}}{\omega}
$$

where $n$ is the refractive index of the unperturbed silicon, $\epsilon_{0}$ is the vacuum permittivity, $\alpha_{\text {in }}$ is the intrinsic absorption coefficient, determined both by the material losses as well as by the optical field scattering at the silicon/silicon oxide interfaces, and $\delta n_{\mathrm{FC}}$ and $\alpha_{\mathrm{FC}}$ are the change in the refractive index and $\mathrm{FC}$ absorption coefficient, respectively. Based on a Drude model of the FCs, the latter two quantities are given by [20]

$$
\begin{aligned}
\delta n_{\mathrm{FC}} & =-\frac{e^{2}}{2 \epsilon_{0} n \omega^{2}}\left[\frac{\Delta N_{e}}{m_{c e}^{*}}+\frac{\left(\Delta N_{h}\right)^{0.8}}{m_{c h}^{*}}\right] \\
\alpha_{\mathrm{FC}} & =\frac{e^{3}}{\epsilon_{0} c n \omega^{2}}\left(\frac{\Delta N_{e}}{\mu_{e} m_{c e}^{*}}+\frac{\Delta N_{h}}{\mu_{h} m_{c h}^{*}}\right)
\end{aligned}
$$

where $e$ is the charge of the electron, $\Delta N_{e}\left(\Delta N_{h}\right)$ is the induced variation of the electrons (holes) density, $m_{c e}^{*}=0.26 m_{0}$ $\left(m_{c h}^{*}=0.39 m_{0}\right)$ is the conductivity effective mass of the electrons (holes), with $m_{0}$ the mass of the electron, and $\mu_{e}\left(\mu_{h}\right)$ is the electron (hole) mobility. Note that in (15) the exponent of the hole density has been introduced for a better fit with the experimental data [20].

The nonlinear part of the polarization $\delta \tilde{\mathbf{P}}(\mathbf{r} ; \omega)$ has two components, namely an electronic part, which contains both the Kerr and the TPA contributions, and the Raman component. The Kerr polarization is the result of the electronic polarizability of silicon, which has a response time of a few tens of femtoseconds, and, therefore, we can assume an instantaneous Kerr response. Moreover, the characteristic time of TPA processes is much shorter than the duration of the pulses considered here, so that we can treat them as instantaneous processes as well. Under these circumstances, the electronic polarization $\delta \tilde{\mathbf{P}}_{e}(\mathbf{r} ; \omega)$ can be written as

$$
\begin{aligned}
\delta \tilde{\mathbf{P}}_{e}(\mathbf{r} ; \omega)=3 \epsilon_{0}\{[ & \hat{\chi}_{p} \mathbf{E}\left(\mathbf{r}, t ; \omega_{p}\right) \mathbf{E}^{*}\left(\mathbf{r}, t ; \omega_{p}\right) \\
& \left.+2 \hat{\chi}_{\mathrm{sp}} \mathbf{E}\left(\mathbf{r}, t ; \omega_{s}\right) \mathbf{E}^{*}\left(\mathbf{r}, t ; \omega_{s}\right)\right] \\
& \times \mathbf{E}\left(\mathbf{r}, t ; \omega_{p}\right) \delta\left(\omega-\omega_{p}\right) \\
+ & {\left[2 \hat{\chi}_{p s} \mathbf{E}\left(\mathbf{r}, t ; \omega_{p}\right) \mathbf{E}^{*}\left(\mathbf{r}, t ; \omega_{p}\right)\right.} \\
& \left.\quad+\hat{\chi}_{s} \mathbf{E}\left(\mathbf{r}, t ; \omega_{s}\right) \mathbf{E}^{*}\left(\mathbf{r}, t ; \omega_{s}\right)\right] \\
& \left.\times \mathbf{E}\left(\mathbf{r}, t ; \omega_{s}\right) \delta\left(\omega-\omega_{s}\right)\right\}+ \text { c.c. }
\end{aligned}
$$

where $\hat{\chi}_{j}=\hat{\chi}^{e}\left(\omega_{j} ; \omega_{j},-\omega_{j}, \omega_{j}\right)$, with $j=p, s$, and $\hat{\chi}_{\mathrm{sp}}=$ $\hat{\chi}^{e}\left(\omega_{p} ; \omega_{s},-\omega_{s}, \omega_{p}\right)$ and $\hat{\chi}_{p s}=\hat{\chi}^{e}\left(\omega_{s} ; \omega_{p},-\omega_{p}, \omega_{s}\right)$ are thirdorder tensor susceptibilities. The real part of these nonlinear susceptibilities describes parametric processes of photon scattering and leads to a change in the refractive index. On the other hand, the imaginary part of the susceptibilities corresponds to TPA processes. Note that since silicon belongs to the crystallographic point group $m 3 m$ the susceptibility tensor $\hat{\chi}^{e}$ has 21 nonzero elements, of which only four are independent, namely $\chi_{1111}^{e}, \chi_{1122}^{e}, \chi_{1212}^{e}$, and $\chi_{1221}^{e}$ [21]. In addition, in the frequency range considered here the frequency dispersion of the nonlinear susceptibility can be neglected, so that the Kleinman symmetry relations imply that the last three elements are equal. The real parts of the two independent susceptibilities are related by $\chi_{1122}^{\prime e}=0.48 \chi_{1111}^{\prime e}[22]$, with $\chi_{1111}^{\prime e}=5.21 \cdot 10^{-19} \mathrm{~m}^{2} \cdot \mathrm{V}^{-2}$ [23], [24].

To find the imaginary parts of the two independent susceptibilities we used the values of the TPA coefficients measured at 
the wavelength $\lambda=1.54 \mu \mathrm{m}$, for the $\langle 110\rangle$ and $\langle 111\rangle$ polarizations [24]. Thus, the TPA coefficient can be expressed as

$$
\beta=\frac{3 \omega}{2 \epsilon_{0} c^{2} n^{2}} \chi_{\mathrm{eff}}^{\prime \prime}
$$

where for silicon

$$
\begin{aligned}
\chi_{\mathrm{eff}}= & \chi_{1122}^{e}\left[\left(\hat{\mathbf{a}}^{*} \cdot \hat{\mathbf{b}}\right)(\hat{\mathbf{c}} \cdot \hat{\mathbf{d}})+\left(\hat{\mathbf{a}}^{*} \cdot \hat{\mathbf{c}}\right)(\hat{\mathbf{b}} \cdot \hat{\mathbf{d}})\right. \\
& \left.+\left(\hat{\mathbf{a}}^{*} \cdot \hat{\mathbf{d}}\right)(\hat{\mathbf{b}} \cdot \hat{\mathbf{c}})\right]+\left(\chi_{1111}^{e}-3 \chi_{1122}^{e}\right) \sum_{i=1}^{3} \hat{\mathbf{a}}_{i}^{*} \hat{\mathbf{b}}_{i} \hat{\mathbf{c}}_{i} \hat{\mathbf{d}}_{i} .
\end{aligned}
$$

Here $\hat{\mathbf{a}}$ is a unit vector along the direction of the induced polarization, $\hat{\mathbf{b}}, \hat{\mathbf{c}}$, and $\hat{\mathbf{d}}$ are unit vectors along the polarization direction of the interacting fields, and $\hat{\mathbf{a}}_{i}, \hat{\mathbf{b}}_{i}, \hat{\mathbf{c}}_{i}$, and $\hat{\mathbf{d}}_{i}$ are the direction cosines of these unit vectors. Thus, for the $\langle 110\rangle$ and $\langle 111\rangle$ polarizations the effective susceptibilities are $\chi_{\mathrm{eff}}^{\langle 110\rangle}=$ $\left(\chi_{1111}^{e}+3 \chi_{1122}^{e}\right) / 2$ and $\chi_{\text {eff }}^{\langle 111\rangle}=\left(\chi_{1111}^{e}+6 \chi_{1122}^{e}\right) / 3$, so that by using the values $\beta_{\langle 110\rangle}=0.79 \mathrm{~cm} / \mathrm{GW}$ and $\beta_{\langle 111\rangle}=$ $0.88 \mathrm{~cm} / \mathrm{GW}$ one obtains $\chi_{1111}^{\prime \prime e}=2.68 \cdot 10^{-20} \mathrm{~m}^{2} \cdot \mathrm{V}^{-2}$ and $\chi_{1122}^{\prime \prime e}=1.82 \cdot 10^{-20} \mathrm{~m}^{2} \cdot \mathrm{V}^{-2}$.

Now let us consider the Raman component of the nonlinear polarization, $\delta \tilde{\mathbf{P}}_{\mathrm{R}}(\mathbf{r} ; \omega)$. The most convenient way to describe this polarization is by means of the third-order Raman susceptibility $\hat{\chi}^{R}\left(\omega_{p}-\omega_{s}\right)$ defined as [25]-[28]

$$
\chi_{i j k l}^{R}(\Omega)=\frac{\pi \mathcal{N}_{v}}{3 \hbar} \sum_{\sigma} \frac{\omega_{\sigma}\left(\alpha_{i j, \sigma} \alpha_{k l, \sigma}+\alpha_{i k, \sigma} \alpha_{l j, \sigma}\right)}{\omega_{\sigma}^{2}-\Omega^{2}+2 i \Omega \Delta \omega}
$$

where $\Omega=\omega_{p}-\omega_{s}, \mathcal{N}_{v}$ is the number of vibrational oscillators per unit volume, $\omega_{\sigma}$ is the vibrational resonant frequency, and the matrix elements $\alpha_{i j, \sigma}$ are given by the derivatives of the polarizability tensor $\hat{\alpha}$ with respect to the coordinate of the normal mode $\sigma$. There are three degenerate Raman-active optical phonons in silicon, $\omega_{\sigma}=\omega_{R}, \sigma=1,2,3$, with the symmetry of the irreducible representation $\Gamma_{25^{\prime}}$, and the corresponding Raman tensors [29]

$$
\mathbf{R}_{1}=\left(\begin{array}{ccc}
0 & 0 & 0 \\
0 & 0 & 1 \\
0 & 1 & 0
\end{array}\right) ; \mathbf{R}_{2}=\left(\begin{array}{ccc}
0 & 0 & 1 \\
0 & 0 & 0 \\
1 & 0 & 0
\end{array}\right) ; \mathbf{R}_{3}=\left(\begin{array}{ccc}
0 & 1 & 0 \\
1 & 0 & 0 \\
0 & 0 & 0
\end{array}\right)
$$

This property implies that there is only one independent component of the Raman tensor, namely $\chi_{1212}^{R}$. Furthermore, close to the Raman resonance $\Omega \simeq \omega_{R}$ so that (19) can be simplified to

$$
\chi_{i j k l}^{R}(\Omega)=\frac{\pi \mathcal{N}_{v}}{6 \hbar} \sum_{\sigma} \frac{\alpha_{i j, \sigma} \alpha_{k l, \sigma}+\alpha_{i k, \sigma} \alpha_{l j, \sigma}}{\omega_{R}-\Omega+i \Delta \omega} .
$$

The real part of this susceptibility describes parametric processes that lead to a change in the refractive index whereas the imaginary part of the Raman susceptibility describes the Raman amplification process. At resonance, $\Omega=\omega_{R}$, the Raman susceptibility is pure imaginary, its value being $\chi_{1212}^{R}\left(\Omega=\omega_{R}\right) \equiv$ $-i \chi_{R}=-i 11.2 \times 10^{-18} \mathrm{~m}^{2} \cdot \mathrm{V}^{-2}$. Under these conditions, and if in addition we assume that the spectral width of the pump and Raman pulses are smaller that the Raman resonance bandwidth, the Raman part of the nonlinear polarization can be written as

$$
\begin{aligned}
\frac{\delta \tilde{\mathbf{P}}_{\mathrm{R}}}{6 \epsilon_{0}}= & \hat{\chi}^{R}\left(-\omega_{R}\right) \mathbf{E}\left(\mathbf{r}, t ; \omega_{s}\right) \mathbf{E}^{*}\left(\mathbf{r}, t ; \omega_{s}\right) \mathbf{E}\left(\mathbf{r}, t ; \omega_{p}\right) \delta\left(\omega-\omega_{p}\right) \\
& +\hat{\chi}^{R}\left(\omega_{R}\right) \mathbf{E}\left(\mathbf{r}, t ; \omega_{p}\right) \mathbf{E}^{*}\left(\mathbf{r}, t ; \omega_{p}\right) \mathbf{E}\left(\mathbf{r}, t ; \omega_{s}\right) \delta\left(\omega-\omega_{s}\right) \\
& + \text { c.c. }
\end{aligned}
$$

Note that since $\hat{\chi}^{R}(\Omega)=\hat{\chi}^{* R}(-\Omega)$ the Raman interaction leads to the amplification of the signal whereas it acts as a loss term at the frequency of the pump.

The expressions (16) and (22) for the nonlinear Kerr and Raman polarization, respectively, as well as the linear polarization described by (14) are inserted in (8) and the result is Fourier transformed into the time domain. As a result, we are left with the following system of coupled partial differential equations, which describe the pulse dynamics upon propagation in the silicon wire waveguide

$$
\begin{aligned}
i \frac{\partial u_{p}}{\partial z} & +i \beta_{1 p} \frac{\partial u_{p}}{\partial t}-\frac{\beta_{2 p}}{2} \frac{\partial^{2} u_{p}}{\partial t^{2}} \\
= & -\frac{i c \beta_{1 p} \kappa_{p}}{2 n}\left(\alpha_{\mathrm{in}}+\alpha_{\mathrm{FC}}^{p}\right) u_{p}-\frac{\omega_{p} \beta_{1 p} \kappa_{p}}{n} \delta n_{\mathrm{FC}}^{p} u_{p} \\
& -\frac{3 \omega_{p} \beta_{1 p}^{2}}{\epsilon_{0} A_{0}}\left(P_{p} \Gamma_{p}\left|u_{p}\right|^{2}+2 P_{s} \Gamma_{\mathrm{sp}}\left|u_{s}\right|^{2}\right) u_{p} \\
& -\frac{6 \omega_{p} \beta_{1 p}^{2} P_{s}}{\epsilon_{0} A_{0}} \Gamma_{R}^{p}\left(-\omega_{R}\right)\left|u_{s}\right|^{2} u_{p} \\
i \frac{\partial u_{s}}{\partial z} & +i \beta_{1 s} \frac{\partial u_{s}}{\partial t}-\frac{\beta_{2 s}}{2} \frac{\partial^{2} u_{s}}{\partial t^{2}} \\
= & -\frac{i c \beta_{1 s} \kappa_{s}}{2 n}\left(\alpha_{\mathrm{in}}+\alpha_{\mathrm{FC}}^{s}\right) u_{s}-\frac{\omega_{s} \beta_{1 s} \kappa_{s}}{n} \delta n_{\mathrm{FC}}^{s} u_{s} \\
& -\frac{3 \omega_{s} \beta_{1 s}^{2}}{\epsilon_{0} A_{0}}\left(P_{s} \Gamma_{s}\left|u_{s}\right|^{2}+2 P_{p} \Gamma_{p s}\left|u_{p}\right|^{2}\right) u_{s} \\
& -\frac{6 \omega_{s} \beta_{1 s}^{2} P_{p}}{\epsilon_{0} A_{0}} \Gamma_{R}^{s}\left(\omega_{R}\right)\left|u_{p}\right|^{2} u_{s}
\end{aligned}
$$

where $A_{0}$ is the transverse area of the wire waveguide and $\Gamma_{p}$, $\Gamma_{s}, \Gamma_{\mathrm{sp}}, \Gamma_{p s}$, and $\Gamma_{R}^{p, s}$ play the role of effective nonlinear susceptibilities, and $\kappa_{p, s}$ are two constants defined as

$$
\kappa_{p, s}=\frac{n^{2} \int_{A_{0}}\left|\mathbf{e}\left(\mathbf{r}_{t} ; \omega_{p, s}\right)\right|^{2} d A}{\int_{A_{\infty}} n^{2}\left(\mathbf{r}_{t}\right)\left|\mathbf{e}\left(\mathbf{r}_{t} ; \omega_{p, s}\right)\right|^{2} d A} .
$$

Although in (24) we kept the frequency dependence of the parameters $\kappa^{\prime}$ s, their frequency dispersion can be neglected. For example, if $\lambda_{p}=1550 \mathrm{~nm}$ and $\lambda_{s}=1670 \mathrm{~nm}$, the corresponding values of these parameters are $\kappa_{p}=0.907$ and $\kappa_{s}=$ 0.871 , respectively. The effective susceptibilities $\Gamma$ are defined by certain weighted integrals of the corresponding tensor susceptibilities $\hat{\chi}$ over the transverse area of the waveguide. Thus, $\Gamma_{p}$ is given by the following expression (we drop the explicit $\mathbf{r}_{t}$-dependence of the modal fields)

$$
\Gamma_{p}=\frac{A_{0} \int_{A_{0}} \mathbf{e}^{*}\left(\omega_{p}\right) \cdot \hat{\chi}_{p}: \mathbf{e}\left(\omega_{p}\right) \mathbf{e}^{*}\left(\omega_{p}\right) \mathbf{e}\left(\omega_{p}\right) d A}{\left(\int_{A_{\infty}} n^{2}\left(\mathbf{r}_{t}\right)\left|\mathbf{e}\left(\omega_{p}\right)\right|^{2} d A\right)^{2}}
$$


the other ones being given by similar formulas, whereas the Raman susceptibilities can be written as

$\Gamma_{R}^{p, s}(\Omega)=\frac{A_{0} \int_{A_{0}} \mathbf{e}^{*}\left(\omega_{p, s}\right) \cdot \hat{\chi}^{R}(\Omega): \mathbf{e}\left(\omega_{s, p}\right) \mathbf{e}^{*}\left(\omega_{s, p}\right) \mathbf{e}\left(\omega_{p, s}\right) d A}{\left(\int_{A_{\infty}} n^{2}\left(\mathbf{r}_{t}\right)\left|\mathbf{e}\left(\omega_{p, s}\right)\right|^{2} d A\right)^{2}}$.

Note that the susceptibility tensors $\hat{\chi}^{e, R}$ are known in the crystal principal axes, whereas the modal fields are numerically determined in the waveguide system. Therefore, to calculate the integrals in (25) and (26) the susceptibility tensors must be transformed first in the waveguide system. For the $(0.45 \mu \mathrm{m}, 0.22 \mu \mathrm{m})$ waveguide, at the wavelengths $\lambda_{p}=1550 \mathrm{~nm}$ and $\lambda_{s}=1670 \mathrm{~nm}$ the corresponding values of the effective susceptibilities are $\Gamma_{p}=\Gamma_{p}^{\prime}+i \Gamma_{p}^{\prime \prime}=7.76 \times 10^{-20}+i 4.97 \times 10^{-21} \mathrm{~m}^{2} \cdot \mathrm{V}^{-2}$, $\Gamma_{s}=6.95 \times 10^{-20}+i 4.45 \times 10^{-21} \mathrm{~m}^{2} \cdot \mathrm{V}^{-2}, \Gamma_{\mathrm{sp}}=$ $7.94 \times 10^{-20}+i 5.09 \times 10^{-21} \mathrm{~m}^{2} \cdot \mathrm{V}^{-2}, \Gamma_{p s}=6.79 \times 10^{-20}+$ $i 4.35 \times 10^{-21} \mathrm{~m}^{2} \cdot \mathrm{V}^{-2}, \Gamma_{R}^{p}\left(-\omega_{R}\right)=i 2.1 \times 10^{-18} \mathrm{~m}^{2} \cdot \mathrm{V}^{-2}$, $\Gamma_{R}^{s}\left(\omega_{R}\right)=-i 1.8 \times 10^{-18} \mathrm{~m}^{2} \cdot \mathrm{V}^{-2}$. These numerical values show that the frequency dispersion of the electronic susceptibilities can be neglected; however, we incorporate in our model the frequency dependence of the Raman susceptibilities $\Gamma_{R}^{p, s}$. The latter are given by the following formula:

$$
\Gamma_{R}^{p, s}(\Omega)=\frac{2 i \omega_{R} \Delta \omega}{\omega_{R}^{2}-\Omega^{2}+2 i \Omega \Delta \omega} \bar{\Gamma}_{R}^{p, s}
$$

where $\bar{\Gamma}_{R}^{p}=-\Gamma_{R}^{p}\left(-\omega_{R}\right)$ and $\bar{\Gamma}_{R}^{s}=\Gamma_{R}^{s}\left(\omega_{R}\right)$. Finally, we mention that in order to derive the system (23) we used the relationship between the total energy density per unit length of the waveguide and the modal optical power, namely $v_{g}=$ $1 / \beta_{1}=P / \mathcal{W}$ where the power $P$ is defined by (7) and $\mathcal{W}=$ $\left(\epsilon_{0} / 2\right) \int_{A_{\infty}} n^{2}\left(\mathbf{r}_{t}\right)\left|\mathbf{e}\left(\mathbf{r}_{t}\right)\right|^{2} d A[14]$.

One important parameter that characterizes the Raman amplification process is the Raman gain coefficient $g_{R}$. By using the equation that governs the dynamics of the amplitude of the Stokes signal, namely the (23b), one can easily show that the Raman gain coefficient is given by the following expression:

$$
g_{R}=\frac{12 \omega_{s} \beta_{1 s}^{2}}{\epsilon_{0}}\left|\bar{\Gamma}_{R}^{s}\right|
$$

Note that since the Raman susceptibility in silicon is about four orders of magnitude larger that the Raman susceptibility of silica, one expects that the Raman amplification in silicon wire is significantly enhanced as compared to the Raman amplification in optical fibers.

For a full description of optical pulse interaction in a silicon wire waveguide the system (23) must be completed with an additional relation that describes the dynamics of the FCs and their interaction with the optical field. For this we use a rate equation, which governs the interplay between the optical FC generation and their decay through recombination processes. Thus, from the system (23) one can easily derive the optical power absorption per unit length due to TPA

$$
\begin{aligned}
\left.\frac{\partial P}{\partial z}\right|_{\mathrm{TPA}}= & -\frac{6}{\epsilon_{0} A_{0}}\left[\omega_{p} \beta_{1 p}^{2}\left(P_{p}^{2} \Gamma_{p}^{\prime \prime}\left|u_{p}\right|^{2}+2 P_{p} P_{s} \Gamma_{\mathrm{sp}}^{\prime \prime}\left|u_{s}\right|^{2}\right)\left|u_{p}\right|^{2}\right. \\
& \left.+\omega_{s} \beta_{1 s}^{2}\left(P_{s}^{2} \Gamma_{s}^{\prime \prime}\left|u_{s}\right|^{2}+2 P_{p} P_{s} \Gamma_{p s}^{\prime \prime}\left|u_{p}\right|^{2}\right)\left|u_{s}\right|^{2}\right]
\end{aligned}
$$

where $P=\left|u_{p}\right|^{2} P_{p}+\left|u_{s}\right|^{2} P_{s}$ is the total optical power. With this expression for the TPA, the rate equation can be written as

$$
\begin{aligned}
\frac{\partial \Delta N}{\partial t}=- & \frac{\Delta N}{t_{c}}+\frac{6}{\epsilon_{0} \hbar A_{0}^{2}}\left[\frac{\beta_{1 p}^{2} P_{p}^{2} \Gamma_{p}^{\prime \prime}}{2}\left|u_{p}\right|^{4}+\frac{\beta_{1 s}^{2} P_{s}^{2} \Gamma_{s}^{\prime \prime}}{2}\left|u_{s}\right|^{4}\right. \\
& \left.+2 P_{p} P_{s} \frac{\omega_{p} \beta_{1 p}^{2} \Gamma_{\mathrm{sp}}^{\prime \prime}+\omega_{s} \beta_{1 s}^{2} \Gamma_{p s}^{\prime \prime}}{\omega_{p}+\omega_{s}}\left|u_{p}\right|^{2}\left|u_{s}\right|^{2}\right]
\end{aligned}
$$

where the characteristic lifetime of the FCs, $t_{c}$, is assumed to be the same for both electrons and holes. In bulk silicon or in waveguides of micrometer size transverse dimensions this characteristic time is a few tens of nanoseconds. However, recently it has been reported that in submicron silicon structures, due to the fast diffusion of free carriers to the edges of the waveguide, this relaxation time can be as low as $0.5 \mathrm{~ns}$ [30].

A better understanding of the interplay between different effects described by the system (23) and (30) is gain if we cast these equations in dimensionless form. For this, we introduce the dimensionless time $\tau=\left(t-z / v_{g p}\right) / T_{0 p}$ and the dimensionless distance $\zeta=z / L_{R}$, where the characteristic Raman length $L_{R}$ is defined by

$$
L_{R}=\frac{\epsilon_{0} A_{0}}{6 \omega_{s} \beta_{1 s}^{2} P_{p}\left|\bar{\Gamma}_{R}^{s}\right|} .
$$

We choose to use the Raman length $L_{R}$ to normalize the distance because for the power levels and transverse waveguide areas used in experiments [3] this characteristic length is the smallest one. For example, for a peak pump power $P_{p}=1 \mathrm{~mW}$, this Raman length is $L_{R}=0.38 \mathrm{~mm}$, which is much smaller than the walkoff length $L_{w}$ or dispersion length $L_{d}$ that we provided before. After simple transformations, the system (23) can be brought to the following dimensionless form:

$$
\begin{aligned}
i \frac{\partial u_{p}}{\partial \zeta} & +\frac{\delta_{p}}{2} \frac{\partial^{2} u_{p}}{\partial \tau^{2}} \\
= & -i \vartheta_{p}\left(\alpha_{\mathrm{in}}+\alpha_{\mathrm{FC}}^{p}\right) u_{p}-\eta_{p} \delta n_{\mathrm{FC}}^{p} u_{p} \\
& -\left(\gamma_{p}\left|u_{p}\right|^{2}+2 \gamma_{\mathrm{sp}}\left|u_{s}\right|^{2}\right) u_{p}-\gamma_{R}^{p}\left|u_{s}\right|^{2} u_{p} \\
i \frac{\partial u_{s}}{\partial \zeta} & +i \nu \frac{\partial u_{s}}{\partial \tau}+\frac{\delta_{s}}{2} \frac{\partial^{2} u_{s}}{\partial \tau^{2}} \\
= & -i \vartheta_{s}\left(\alpha_{\mathrm{in}}+\alpha_{\mathrm{FC}}^{s}\right) u_{s}-\eta_{s} \delta n_{\mathrm{FC}}^{s} u_{s} \\
& -\left(\gamma_{s}\left|u_{s}\right|^{2}+2 \gamma_{p s}\left|u_{p}\right|^{2}\right) u_{s}-\gamma_{R}^{s}\left|u_{p}\right|^{2} u_{s}
\end{aligned}
$$

where the parameters in (32) are given by the following expressions:

$$
\begin{aligned}
\nu & =\operatorname{sgn}\left(d_{p s}\right) \frac{L_{R}}{L_{w}} \quad \delta_{p}=-\frac{\operatorname{sgn}\left(\beta_{2 p}\right) L_{R}}{L_{d}} \quad \delta_{s}=\frac{\delta_{p} \beta_{2 s}}{\beta_{2 p}} \\
\vartheta_{p} & =\frac{c \beta_{1 p} \kappa_{p} L_{R}}{2 n} \quad \vartheta_{s}=\frac{\vartheta_{p} \beta_{1 s} \kappa_{s}}{\beta_{1 p} \kappa_{p}} \quad \eta_{p, s}=\frac{2 \vartheta_{p, s} \omega_{p, s}}{c} \\
\gamma_{p} & =\frac{\omega_{p} \beta_{1 p}^{2} \Gamma_{p}}{2 \omega_{s} \beta_{1 s}^{2}\left|\bar{\Gamma}_{R}^{s}\right|} \quad \gamma_{s}=\frac{P_{s} \Gamma_{s}}{2 P_{p}\left|\bar{\Gamma}_{R}^{s}\right|} \quad \gamma_{p s}=\frac{\Gamma_{p s}}{\left|\bar{\Gamma}_{R}^{s}\right|} \\
\gamma_{\mathrm{sp}} & =\frac{\omega_{p} \beta_{1 p}^{2} P_{s} \Gamma_{\mathrm{sp}}}{\omega_{s} \beta_{1 s}^{2} P_{p}\left|\bar{\Gamma}_{R}^{s}\right|} \quad \gamma_{R}^{p}=-\frac{\omega_{p} \beta_{1 p}^{2} P_{s} \bar{\Gamma}_{R}^{p}}{\omega_{s} \beta_{1 s}^{2} P_{p}\left|\bar{\Gamma}_{R}^{s}\right|} \\
\gamma_{R}^{s} & =\frac{\bar{\Gamma}_{R}^{s}}{\left|\bar{\Gamma}_{R}^{s}\right|} .
\end{aligned}
$$


Similarly, in the dimensionless variables the rate equation (30) can be written as

$$
\frac{\partial \Delta N}{\partial \tau}=-\frac{\Delta N}{\tau_{c}}+\rho_{p}\left|u_{p}\right|^{4}+\rho_{s}\left|u_{s}\right|^{4}+\left(\rho_{\mathrm{sp}}+\rho_{p s}\right)\left|u_{p}\right|^{2}\left|u_{s}\right|^{2}
$$

where $\tau_{c}=t_{c} / T_{0 p}$ is the normalized FC relaxation time and

$\rho_{p, s}=\frac{3 T_{0 p} \beta_{1 p, s}^{2} P_{p, s}^{2} \Gamma_{p, s}^{\prime \prime}}{\epsilon_{0} \hbar A_{0}^{2}} \rho_{s p, p s}=\frac{12 T_{0 p} \omega_{p, s} \beta_{1 p, s}^{2} P_{p} P_{s} \Gamma_{s p, p s}^{\prime \prime}}{\epsilon_{0} \hbar A_{0}^{2}\left(\omega_{p}+\omega_{s}\right)}$.

Note that in (32) $\delta n_{\mathrm{FC}}^{p, s}$ and $\alpha_{\mathrm{FC}}^{p, s}$ depend on the FC concentration $\Delta N$ through (15) and, therefore, (32) and (34) must be solved simultaneously.

When the spectral width of the pump and Stokes pulse is comparable or larger than the width of the Raman spectrum $\Delta \omega$ the system (32) must be modified so as to take into account the finite time response of the SRS process. To this end, we first introduce the Raman response function in the wire waveguide $G_{R}(t)$, defined as the inverse Fourier transform of the Raman effective susceptibility $\Gamma_{R}(\Omega)$

$$
\begin{aligned}
G_{R}^{p, s}(t) & =\frac{1}{2 \pi} \int_{-\infty}^{\infty} \Gamma_{R}^{p, s}(\Omega) e^{i \Omega t} d t \\
& =\frac{2 i \omega_{R} \Delta \omega}{\bar{\omega}_{R}} \bar{\Gamma}_{R}^{p, s} e^{-\Delta \omega t} \mathcal{H}(t) \sin \left(\bar{\omega}_{R} t\right)
\end{aligned}
$$

where $\bar{\omega}_{R}=\sqrt{\omega_{R}^{2}-\Delta \omega^{2}}$ and $\mathcal{H}(t)$ is the Heaviside function defined as $\mathcal{H}(t)=1$ for $t>0$ and $\mathcal{H}(t)=0$ for $t<0$. In order to include in our formalism the Raman delay described by the response function (36), we follow the approach developed in [12], [31]-[33]. As the derivation is similar to that presented in these works, we give here only the final form of the governing equations:

$$
\begin{aligned}
i \frac{\partial u_{p}}{\partial \zeta} & +\frac{\delta_{p}}{2} \frac{\partial^{2} u_{p}}{\partial \tau^{2}} \\
= & -i \vartheta_{p}\left(\alpha_{\mathrm{in}}+\alpha_{\mathrm{FC}}^{p}\right) u_{p}-\eta_{p} \delta n_{\mathrm{FC}}^{p} u_{p} \\
& -\left(\gamma_{p}\left|u_{p}\right|^{2}+2 \gamma_{\mathrm{sp}}\left|u_{s}\right|^{2}\right) u_{p}-\gamma_{R}^{p} u_{s}(\zeta, \tau) \\
& \times \int_{-\infty}^{\infty} g_{R}^{p}\left(\tau-\tau^{\prime}\right) u_{s}^{*}\left(\zeta, \tau^{\prime}\right) u_{p}\left(\zeta, \tau^{\prime}\right) \\
& \times e^{i \tilde{\omega}_{R}\left(\tau-\tau^{\prime}\right)} d \tau^{\prime} \\
i \frac{\partial u_{s}}{\partial \zeta} & +i \nu \frac{\partial u_{s}}{\partial \tau}+\frac{\delta_{s}}{2} \frac{\partial^{2} u_{s}}{\partial \tau^{2}} \\
= & -i \vartheta_{s}\left(\alpha_{\mathrm{in}}+\alpha_{\mathrm{FC}}^{s}\right) u_{s}-\eta_{s} \delta n_{\mathrm{FC}}^{s} u_{s} \\
& -\left(\gamma_{s}\left|u_{s}\right|^{2}+2 \gamma_{p s}\left|u_{p}\right|^{2}\right) u_{s}-\gamma_{R}^{s} u_{p}(\zeta, \tau) \\
& \times \int_{-\infty}^{\infty} g_{R}^{s}\left(\tau-\tau^{\prime}\right) u_{p}^{*}\left(\zeta, \tau^{\prime}\right) u_{s}\left(\zeta, \tau^{\prime}\right) \\
& \times e^{-i \tilde{\omega}_{R}\left(\tau-\tau^{\prime}\right)} d \tau^{\prime}
\end{aligned}
$$

where $\tilde{\omega}_{R}=\omega_{R} T_{0 p}$ is the dimensionless Raman frequency and $g_{R}^{p, s}(\tau)=T_{0 p} G_{R}^{p, s}\left(T_{0 p} \tau\right) / \bar{\Gamma}_{R}^{p, s}$ is the normalized Raman response function. Simple calculations show that when the spectral width of the pulses $u_{p}(\zeta, \tau)$ and $u_{s}(\zeta, \tau)$ is much smaller than $\Delta \omega$ the system (37) reduces to (32). Therefore, to find the dynamics of picosecond pulses as they interact through
SRS one has to numerically integrate (37) coupled to the rate equation (34).

\section{RAMAN AMPLIFICATION AND PULSE INTERACTIONS}

In this section we numerically investigate the physical characteristics of the Raman pulse interaction in silicon wires. We discuss both noise-seeded Stokes signal generation and amplification as well as Raman amplification of input signal pulses. In both cases we investigate the Raman amplification of pulses with duration much longer than the Raman response time, which can be thought of as a quasi-continuous-wave $(\mathrm{CW})$ regime, as well as the dynamics of pulses with duration comparable or shorter than the Raman response time. We compare the predictions of our theoretical model with experimental data and find good agreement. We also discuss potential applications to optoelectronics nanodevices.

The numerical simulations of the coupled mode equations (32) [or (37)] were carried out by using the split-step fast Fourier transform method (for details, see [12]), which assumes that over an integration step $h$ the linear and nonlinear effects act independently. We adopted the symmetrized version of the method, which has improved computational speed. The basic procedure is to allow the pulses to propagate half of the step under the influence of the linear terms only, then the nonlinear effect along the whole step is added to the pulses at the middle of the integration step, and finally the pulses are propagated for the second half of the step with only the linear terms included. In our calculation, the size of the time domain window is chosen to be 16 times as large as the pump pulse width, whereas the number of time domain sampling points is 1024 .

To include the free carrier effects, we need to compute the FC density at each propagation step, which is determined by the rate equation (34). Since the rate equation and the coupled mode equations (32) [or (37)] are nonlinearly coupled, we calculated the free carrier density at the middle of each propagation step. The integration of both the nonlinear part of the coupled mode equations as well as the rate equation is performed by using a fifth-order Cash-Karp Runge-Kutta algorithm [34].

We started by considering noise-seeded Raman amplification in the case in which the pump pulse has a temporal width $T_{0 p}=30$ ps. Because this pulsewidth is considerably larger than the Raman response time of silicon, the system can respond effectively instantaneously; the pulse dynamics are then governed by (32). In the SRS process, the critical pump power for amplification is defined as the pump power that is required for the Stokes signal to be amplified to the same intensity as the input pump power [12]. The pump peak power was chosen to be $P_{p}=0.1 \mathrm{~mW}$, which is about two orders of magnitude larger than the critical power. As long as the propagation distance is much larger than the walkoff length $L_{w}$, this critical power is expressed as [12]

$$
\frac{\sqrt{\pi} g_{R} P_{p}^{\mathrm{cr}} L_{w}}{A_{0}}=16
$$

The broad-band noise power is approximately given by the product between the energy of the Stokes photon $\hbar \omega_{s}$ and the Raman gain bandwidth, here we assume $P_{s}=1.0 \times 10^{-8} \mathrm{~W}$. 

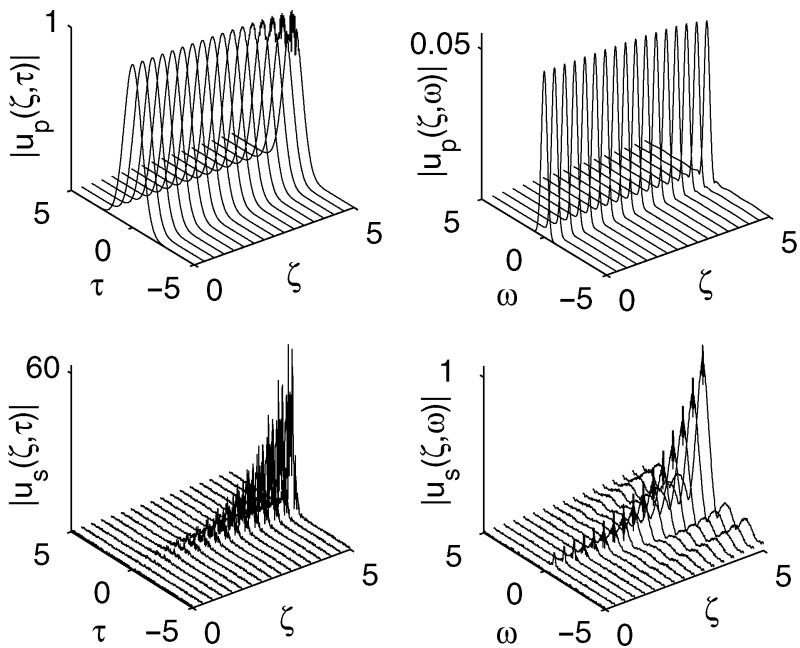

Fig. 3. Noise-seeded Raman generation from a 30-ps pump pulse and no intrinsic losses. The pump power is $P_{p}=0.1 \mathrm{~mW}$, which corresponds to a Raman length $L_{R}=3.83 \mathrm{~mm}$, and the seed-noise power is $P_{s}=1.0 \times 10^{-8} \mathrm{~W}$. The left and right panels show the pulse in the time and frequency domain as it propagates along the waveguide. The units are in the dimensionless forms described in the text, e.g., paragraph above (31) for the definition of $\tau$ and $\zeta$.

In our first calculation we set the intrinsic loss of the waveguide to zero but allow the waveguide to have optical losses due to the FC and TPA processes. The results, presented in Fig. 3, clearly demonstrate coherent generation of the Raman signal. Furthermore, as the optical losses are very small and the seeded energy at the Raman frequency is also relatively low, the pump pulse is undepleted during the initial stage of the pulse propagation. However, after a propagation distance of about $4 L_{R}$ the power in the Raman pulse grows to a level at which its interaction with the pump pulse becomes large enough to change its temporal profile. This change is seen either near the peak of the pump pulse (top figure, left panel) or in the side lobes seen in the bottom of the figure on the right panel.

To illustrate a more realistic case, we considered a waveguide with intrinsic losses of $3.5 \mathrm{~dB} / \mathrm{cm}$, that is equal to the losses of waveguides used in recent experiments [3], whereas the power of the pump pulse was taken to be the same as before, $P_{p}=0.1 \mathrm{~mW}$. Fig. 4 shows that in this case the pump pulse is strongly depleted, as the Raman pulse grows from the seeded noise. In addition, since the waveguide losses are higher, the amplification of the Raman pulse over the same propagation distance is much smaller than the amplification observed in a lossless waveguide.

Because of the stronger pump depletion in the waveguide with intrinsic losses the amount of the FCs generated by TPA is markedly different in the two cases. To illustrate these facts, we present in Fig. 5 the FC density, calculated for different distances along the wire waveguide. As expected, in the regime of strong pump depletion the peak value of the FC density also decreases with the propagation distance. However, Fig. 5 shows that there are similar time dynamics for the FCs generated in the two cases. The figure displays the carrier density $\Delta N$ at different propagation distances $\zeta$ and for each $\zeta$, the carrier density is displayed versus the time in the traveling time coordinate
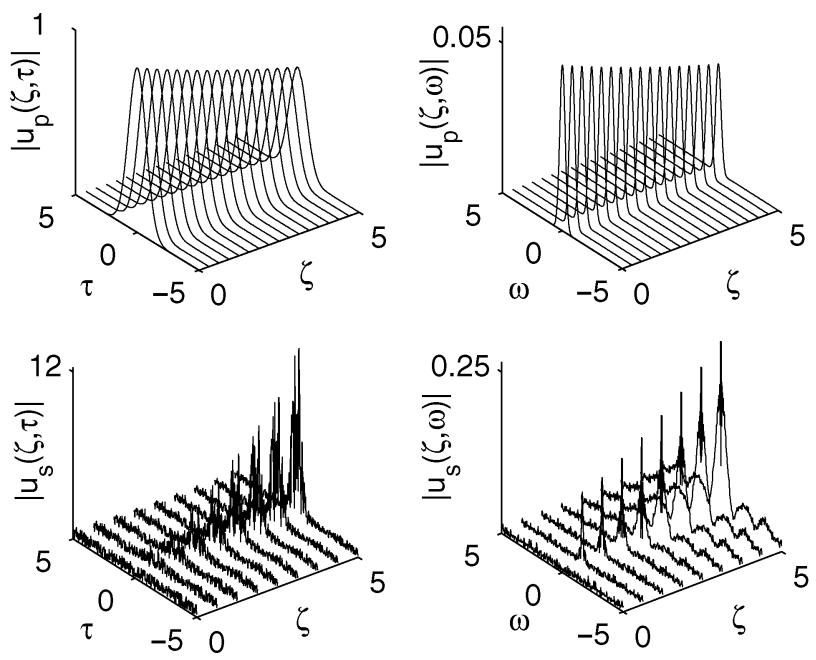

Fig. 4. Results of the same calculation as in Fig. 3 except that a $3.5 \mathrm{~dB} / \mathrm{cm}$ intrinsic loss is added to the waveguide.
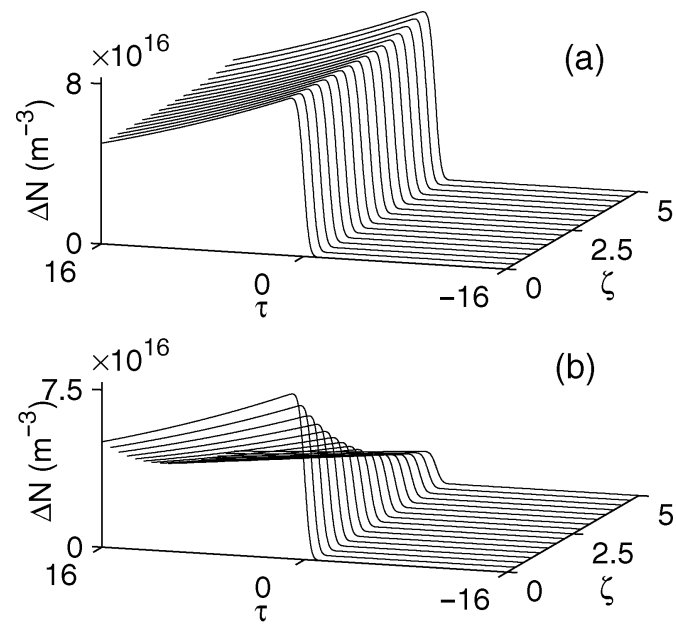

Fig. 5. Carrier density dynamics at different propagation distances. The simulation parameters in (a) and (b) correspond to those in Fig. 3 and Fig. 4, respectively. Note that for the $x$-axis, normalized time, $\tau$, progresses from right to left. The rise time of the FC density (FCD), i.e., the sharp increase, is determined by the temporal width of the pump pulse whereas the subsequent decay time by the characteristic relaxation time $t_{c}$, i.e., the slow decay. Without intrinsic loss (the upper figure), the maximum value of the FCD remains nearly constant along the waveguide. With intrinsic loss (the lower figure), the temporal profile of the FCD is still the same, but its maximum value decreases in the same manner as the pump-pulse intensity.

system. Note that the time increases from right to left. This carrier density rises rapidly with the pulse rise time and decay more slowly as the carrier recombines. This behavior is explained by the fact that the rise time of the FC density is determined by the temporal width of the pump pulse whereas the subsequent decay time by the characteristic relaxation time $t_{c}$; both these characteristic times are independent on the pump power.

We have performed a detailed analysis of the Raman amplification process illustrated in Fig. 3 and Fig. 4. For this, we determined the peak power of the Raman signal that is generated over a certain propagation distance in the wire waveguide as a function of the input peak power of the pump pulse. The calculations were performed both for lossless waveguide as well as for a waveguide with intrinsic losses of 0.5 and $3.5 \mathrm{~dB} / \mathrm{cm}$. For the 


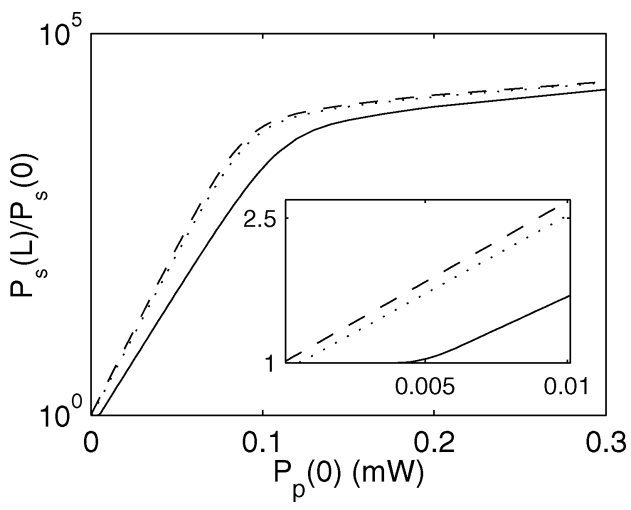

Fig. 6. Raman amplification versus the pump peak power, computed for a lossless silicon waveguide (- - ) and for waveguide losses of $0.5 \mathrm{~dB} / \mathrm{cm}$ $(\cdots)$ and $3.5 \mathrm{~dB} / \mathrm{cm}(-)$. The waveguide length is $L=2 \mathrm{~cm}$. The inset shows a zoom-in of the region of low pump power. Both plots are drawn using semi-logarithm scales.

seed signal pulse we used a Gaussian with the initial amplitude $P_{s}(\zeta=0)=1.0 \times 10^{-8} \mathrm{~W}$ and both the signal and the pump pulse had a temporal width of 30 ps. The data shown in Fig. 6 correspond to a propagation distance of $L=2 \mathrm{~cm}$. This figure demonstrates that for waveguide losses that correspond to those measured in recent experiments [3], a 0.1-mW pump power can amplify a Raman signal from noise to the power level comparable to that of the pump, over a relatively short distance. Also, one observes that the amplification process begins to saturate at lower pump powers if the waveguide losses are decreased. Finally, the inset in Fig. 6 shows that, as expected, the threshold pump power required to observe the Raman amplification of the signal increases with the intrinsic optical losses in the wire waveguide.

A different Raman pulse interaction regime is that in which the input pump and the seeded Raman signal have comparable peak powers. The interaction of such pulses, as well as the corresponding dynamics of the generated FCs is illustrated in Fig. 7. Note that unlike the case of small power levels of the seeded signal pulse, see Fig. 4, when the powers of the pump and signal pulses are comparable, the pump pulse is not only strongly depleted but its temporal profile is also markedly reshaped. Thus, upon a propagation distance of $5 L_{R}$ the central part of the pump pulse is almost completely depleted so that at the output facet of the waveguide it emerges at two temporally separated pulses. The dynamics of the signal pulse also shows interesting features, namely initially it is amplified over a certain distance and then, when the losses compensate the Raman amplification, it starts to decay. From a practical point of view, this suggests that there is an optimum distance at which the signal amplification reaches a maximum value. Note also that, as expected, in this case of high signal pulse power the amount of generated FCs is considerably increased.

When the temporal duration of the interacting pulses is comparable to the Raman response time of silicon (a few picoseconds) the effect of the delayed response of the nonlinear medium must be incorporated into the model, so that (37) must be used to describe the dynamics of the optical pulses. In addition, for such short pulses the walkoff length is comparable to the length of the waveguides used in practical applications and, therefore,
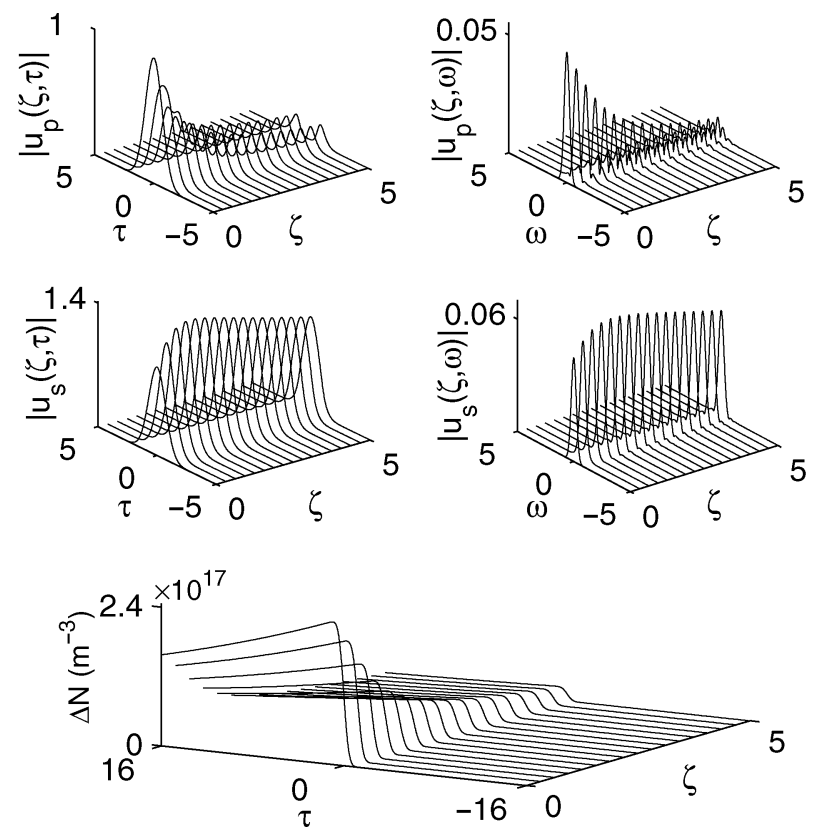

Fig. 7. Raman interaction of a pump and a signal pulse, in a silicon wire waveguide with $3.5 \mathrm{~dB} / \mathrm{cm}$ intrinsic optical losses, both pulses having a 30-ps temporal width. The pump power is $P_{p}=0.1 \mathrm{~mW}$, which corresponds to a Raman length $L_{R}=3.83 \mathrm{~mm}$, and the seed signal power is $P_{s}=0.05 \mathrm{~mW}$. The lowest panel shows the dynamics of the optically generated FCs; see the caption of Fig. 5 for general comments on plot used in this panel.

the pulse walkoff plays an important role. To illustrate this, we present in Fig. 8 the dynamics of the interaction of a pump and a signal pulse with the temporal width of $2 \mathrm{ps}$, for which the corresponding walkoff length is $L_{w}=4.76 \mathrm{~cm}$, as they propagate a distance $L=16 L_{R}=6.13 \mathrm{~cm}$. Thus, Fig. 8 shows that the group velocity mismatch between the two pulses reduces they effective interaction length and, therefore, it limits the efficiency of the Raman amplification. Furthermore, because of the temporal walkoff between the two pulses, the temporal profile of the two pulses, at the output facet of the waveguide, is strongly asymmetric. In addition, the pump pulse self-induces a significant large temporal modulation, which is seen clearly in Fig. 8 bottom left panel. This modulation appears as a wide plateau in the pump spectrum.

It is desirable to compare the output of our theoretical model with that measured experimentally. Unfortunately, a set of complete experimental data does not exist. However, some guidance can be obtained using results from other larger waveguides. The highest Raman amplification in silicon waveguides reported so far is from [35]. In this case, $24 \mathrm{~dB}$ intrinsic Raman gain was achieved using 50-ps pulses in a 2.5 -cm silicon waveguide with a large cross section of $2 \mu \mathrm{m}^{2}$. The peak power of the pump pulse was $39 \mathrm{~W}$. By comparison, if we apply a 50-ps pump pulse with the same power density to the silicon wire waveguide, e.g., the peak power of pump at $2 \mathrm{~W}$, our model predicts an intrinsic Raman gain of about $33 \mathrm{~dB}$. Considering that these two waveguides have different dispersion properties, different TPA characteristics and different mode-matching conditions, plus the fact that the pump pulses used in these two cases are likely to have different spectral contents, the prediction from our model is well within the acceptable agreement range with the experimental results. 

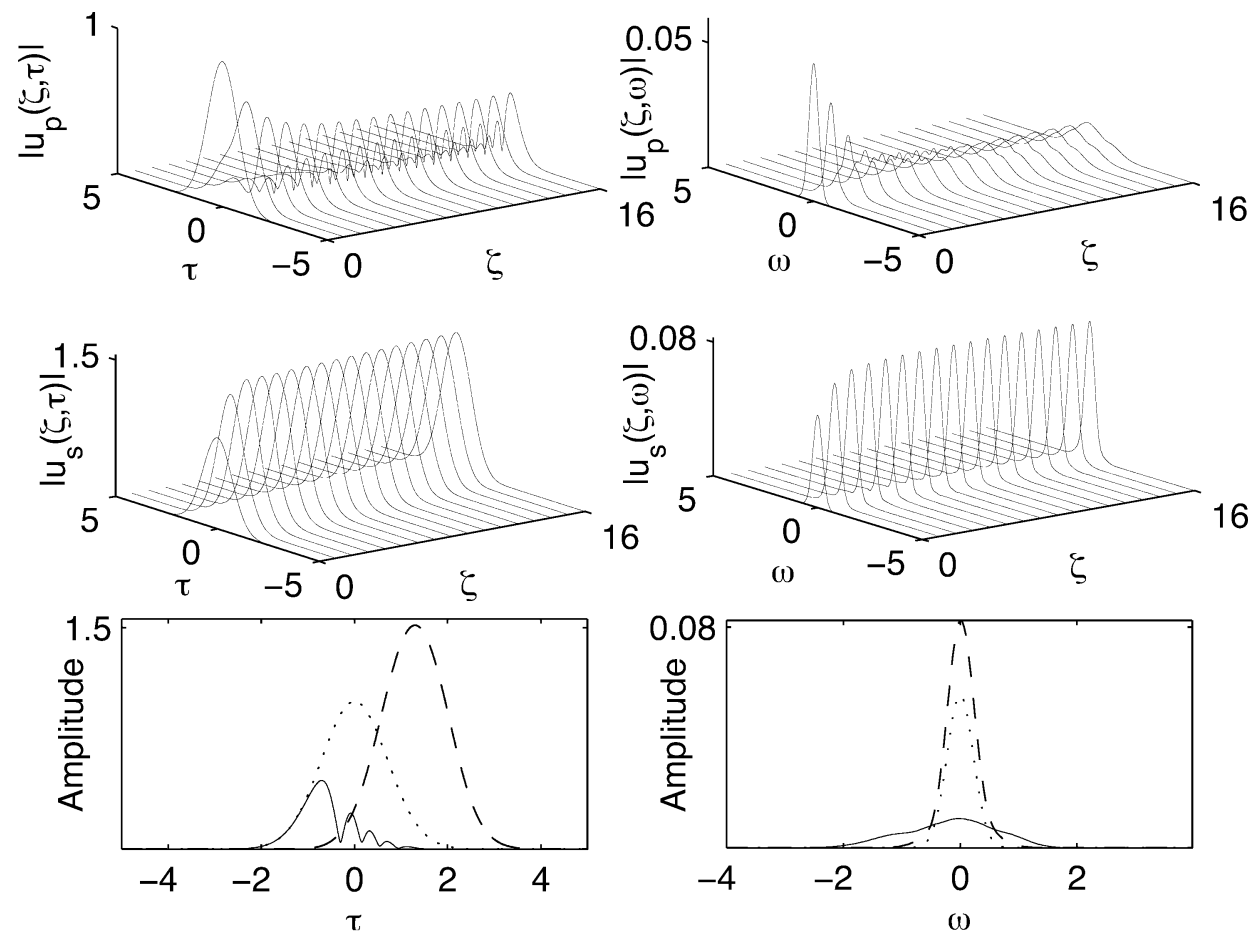

Fig. 8. Raman interaction of a pump and a signal pulse, in a lossless silicon wire waveguide, both pulses having a 2-ps temporal width (the walkoff length is $L_{w}=4.76 \mathrm{~cm}$ ). The pump power is $P_{p}=0.1 \mathrm{~mW}$, which corresponds to a Raman length $L_{R}=3.83 \mathrm{~mm}$, and the seed signal power is $P_{s}=0.05 \mathrm{~mW}$. The lowest panels show the input profile of both pulses (dotted line) and the output profile of the pump (continuous line) and the Raman signal (dashed line), both in the time (left panel) and frequency (right panel) domains. The waveguide in this case is $L=16 L_{R}=6.13 \mathrm{~cm}$.

\section{CONCLUSION}

We have presented a comprehensive analysis of pulsed Raman amplification in silicon wire waveguides. By using the coupled mode analysis we have first developed a theoretical model of the Raman interaction of optical pulses upon propagation in a wire waveguide, which includes both linear effects such as intrinsic waveguide optical losses, phase shift and losses due to FCs generated through TPA, or first- and second-order frequency dispersion as well as nonlinear effects, which includes SPM and XPM, TPA losses, and the interpulse Raman interaction. The dynamics of the FCs generated through TPA has also been incorporated by means of a rate equation, which is coupled to the system of equations that describes the dynamics of the two-frequency optical field. The theoretical model we have developed describes both the interaction of pulses whose temporal width is much larger than the response time of the Raman medium (the quasi-CW regime), as well as the interaction of pulses with the temporal width comparable or smaller than the Raman response time.

The system of coupled equations that describes dynamics of both the optical field and the corresponding FCs generated through TPA has subsequently been numerically integrated, and phenomena such as noise-seeded Raman amplification, pulsed Raman amplification, and Raman-mediated pulse interaction have been described. Finally, we mention the that analytical model introduced here can be adapted to study the optical properties of more complex silicon-based optoelectronic devices, such as ring modulators or Raman lasers.

\section{ACKNOWLEDGMENT}

The authors thank Dr. Jerry I. Dadap and Dr. Richard L. Espinola for helpful discussion. We also wish to thank Dr. Yurii A. Vlasov for his useful comments.

\section{REFERENCES}

[1] R. Claps, D. Dimitropoulos, V. Raghunathan, Y. Han, and B. Jalali, "Observation of stimulated Raman amplification in silicon waveguides," Opt. Exp., vol. 11, pp. 1731-1739, 2003.

[2] D. Dimitropoulos, B. Houshmand, R. Claps, and B. Jalali, "Coupledmode theory of Raman effect in silicon-on-insulator waveguides," Opt. Lett., vol. 28, pp. 1954-1956, 2003.

[3] R. L. Espinola, J. I. Dadap, R. M. Osgood, S. J. McNab, and Y. A. Vlasov, "Raman amplification in ultrasmall silicon-on-insulator wire waveguides," Opt. Exp., vol. 12, pp. 3716-3718, 2004.

[4] J. I. Dadap, R. L. Espinola, R. M. Osgood, S. J. McNab, and Y. A. Vlasov, "Spontaneous Raman scattering in ultrasmall silicon waveguides," Opt. Lett., vol. 29, pp. 2755-2757, 2004.

[5] O. Boyraz and B. Jalali, "Demonstration of a silicon Raman laser," Opt Exp., vol. 12, pp. 5269-5273, 2004.

[6] - "Demonstration of directly modulated silicon Raman laser," Opt. Exp., vol. 13, pp. 796-800, 2005.

[7] H. Rong, A. Liu, R. Jones, O. Cohen, D. Hak, R. Nicolaescu, A. Fang, and M. Paniccia, "An all-silicon Raman laser," Nature, vol. 433, pp. 294-296, 2005.

[8] H. Rong, R. Jones, A. Liu, O. Cohen, D. Hak, R. Nicolaescu, A. Fang, and M. Paniccia, "A continuous-wave Raman silicon laser," Nature, vol. 433, pp. 725-728, 2005.

[9] R. Claps, V. Raghunathan, D. Dimitropoulos, and B. Jalali, “Anti-stokes Raman conversion in silicon waveguides," Opt. Exp., vol. 11, pp. 2862-2872, 2003.

[10] R. L. Espinola, J. I. Dadap, R. M. Osgood Jr., S. J. McNab, and Y. A Vlasov, "C-band wavelength conversion in silicon photonic wire waveguides," Opt. Exp., vol. 13, pp. 4341-4349, 2005.

[11] E. D. Palik, Handbook of Optical Constants of Solids. San Diego, CA: Academic, 1998. 
[12] G. P. Agrawal, Nonlinear Fiber Optics. San Diego, CA: Academic, 2001.

[13] P. A. Temple and C. E. Hathaway, "Multiphonon Raman spectrum of silicon," Phys. Rev. B, vol. 7, pp. 3685-3697, 1973.

[14] A. W. Snyder and J. D. Love, Optical Waveguide Theory. London, U.K.: Chapman Hall, 1983.

[15] A. Hardy and W. Streifer, "Coupled mode theory of parallel waveguides," J. Lightw. Technol., vol. LT-3, no. 5, pp. 1135-1146, Oct. 1985.

[16] S.-L. Chuang, "A coupled mode formulation by reciprocity and a variational principle," J. Lightw. Technol., vol. LT-5, no. 5, pp. 5-15, Oct. 1987.

[17] H. A. Haus, W. P. Huang, S. Kawakami, and N. A. Whitaker, "Coupledmode theory of optical waveguides," J. Lightw. Technol., vol. LT-5, no. 1, pp. 16-23, Jan. 1987.

[18] A. A. Hardy, "A unified approach to coupled-mode phenomena," IEEE J. Quantum Electron., vol. 34, no. 7, pp. 1109-1116, Jul. 1998.

[19] A. N. Kireev and T. Graf, "Vector coupled-mode theory of dielectric waveguides," IEEE J. Quantum Electron., vol. 39, no. 7, pp. 866-873, Jul. 2003.

[20] R. A. Soref and B. R. Bennett, "Electrooptical effects in silicon," IEEE J. Quantum Electron., vol. 23, no. 1, pp. 123-129, Jan. 1987.

[21] R. W. Boyd, Nonlinear Optics. San Diego, CA: Academic, 2003.

[22] J. J. Wynne, "Optical third-order mixing in GaAs, Ge, Si, and InAs," Phys. Rev., vol. 178, pp. 1295-1303, 1969.

[23] H. K. Tsang, C. S. Wong, T. K. Liang, I. E. Day, S. W. Roberts, A. Harpin, J. Drake, and M. Asghari, "Optical dispersion, two-photon absorption and self-phase modulation in silicon waveguides at $1.5 \mathrm{~mm}$ wavelength," Appl. Phys. Lett., vol. 80, pp. 416-418, 2002.

[24] M. Dinu, F. Quochi, and H. Garcia, "Third-order nonlinearities in silicon at telecom waveguides," Appl. Phys. Lett., vol. 82, pp. 2954-2956, 2003.

[25] Y. R. Shen and N. Bloembergen, "Theory of stimulated Brillouin and Raman scattering," Phys. Rev., vol. 137, pp. A1787-A1805, 1965.

[26] M. D. Levenson and N. Bloembergen, "Dispersion of the nonlinear susceptibility tensor in centrosymmetric media," Phys. Rev. B, vol. 15, pp. 4447-4464, 1974.

[27] M. Cardona, "Resonance phenomena," in Light Scattering in Solids II, M. Cardona and G. Guntherodt, Eds. Berlin, Germany: Springer, 1982, vol. 50, Topics in Applied Physics, ch. 2.

[28] H. Vogt, "Coherent and hyper-Raman techniques," in Light Scattering in Solids II, M. Cardona and G. Guntherodt, Eds. Berlin, Germany: Springer-Verlag, 1982, vol. 50, ch. 4.

[29] R. Loudon, "The Raman effect in crystals," Adv. Phys., vol. 13, pp. 423-480, 1964

[30] P. E. Barclay, K. Srinivasan, and O. Painter, "Nonlinear response of silicon photonic crystal microresonators excited via an integrated waveguide and fiber taper," Opt. Exp., vol. 13, pp. 801-820, 2005.

[31] A. Hook, "Influence of stimulated Raman scattering on cross-phase modulation between waves in optical fibers," Opt. Lett., vol. 17, pp. $115-117,1992$.

[32] F. X. Kartner, D. J. Dougherty, H. A. Haus, and E. P. Ippen, "Raman noise and soliton squeezing," J. Opt. Soc. Amer. B, vol. 11, pp. 1267-1276, 1994.

[33] C. Headley and G. P. Agrawal, "Unified description of ultrafast stimulated Raman scattering in optical fibers," J. Opt. Soc. Amer. B, vol. 13, pp. 2170-2177, 1996.

[34] W. H. Press, S. A. Teukolsky, W. T. Vetterling, and B. P. Flannery, Numerical Recipes in C. Cambridge, U.K.: Cambridge Univ. Press, 1997.
[35] V. Raghunathan, O. Boyraz, and B. Jalali, "20 dB on-off Raman amplification in silicon waveguides," presented at the CLEO/LEOS/Phast 05, 2005, Paper CMU1.

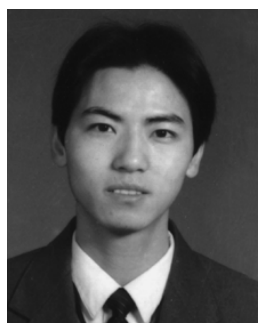

Xiaogang Chen received the B.Sc. and M.S. degrees from the Physics Department, University of Science and Technology of China, Hefei, China, in 1995 and 1997, respectively. He is currently working toward the Ph.D. degree in electrical engineering at Columbia University, New York.

He was a Member of Technical Staff of Lucent Technologies, Inc. from 1999 to 2002. His research interests include nonlinear optics, integrated optics, silicon Raman lasers devices and fiber optical communication systems.

Mr. Chen is a student member of the Optical Society of America and the IEEE Lasers and Electro-Optics Society.

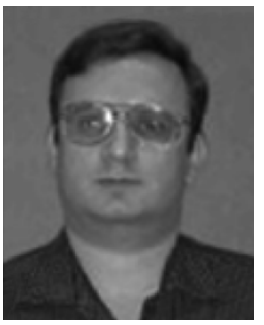

Nicolae C. Panoiu received the B.Sc. and M.S. degrees in physics from the University of Bucharest, Bucharest, Romania, in 1990 and 1992, respectively, and the Ph.D. degree from New York University, New York, in 2001.

$\mathrm{He}$ is currently an Associate Research Scientist with the Department of Applied Physics and Applied Mathematics, Columbia University, New York. His research interests include optical solitons in fibers and quadratic media, photonic crystals, left-handed metamaterials, and electromagnetic structures.

Dr. Panoiu is a member of the Optical Society of America and of the American Physical Society.

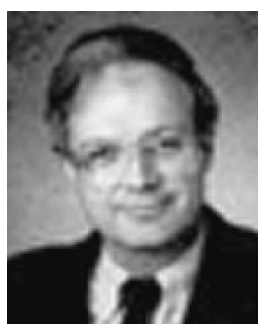

Richard M. Osgood, Jr. (SM'82-F'87) received the B.Sc. degree from the U.S. Military Academy, West Point, NY, the M.S. degree from The Ohio State University, Columbus, and the Ph.D. degree from the Massachusetts Institute of Technology (MIT), Cambridge.

$\mathrm{He}$ is currently the Higgins Professor of Applied Physics and Electrical Engineering at Columbia University, New York. Throughout his professional career, he has performed research in many areas of optical and chemical physics and electrical engineering. His most extensive research has been in the development of new infrared and ultraviolet lasers, and different applications of laser-induced chemistry.

Dr. Osgood is a Member of the American Chemical Society and the Materials Research Society and a Fellow of the Optical Society of America and the American Physical Society. 